NOTA CIENTÍFICA

\title{
Descripción de lesiones pulmonares por Pseudalius inflexus en la marsopa Phocaena spinipinnis
}

\author{
Description of pulmonary lesions by Pseudalius inflexus in the porpoise \\ Phocaena spinipinnis
}

1 Departamento de Salud Animal y Salud Pública, Facultad de Medicina Veterinaria, Universidad Nacional Mayor de San Marcos, Av. Circunvalación s/n. Lima, Perú. Email Alfonso Chavera: 2 Departamento de Ciencias Biológicas, Facultad de Ciencias, Uicas, Facultad de Ciencias, Universidad Gonzaga. Ica, Peru.

3 Dirección actual: Grupo Temático de Enfermedades Metaxénicas, Dirección General de Epidemiología, Ministerio de Salud Calle Danie Ministerio de Salud. Calle Danie Plaechea No 199, Lima 11. Lima, Perú. Email Rufino Cabrera: rcabrerach@hotmail.com 4 Laboratorio de Parasitología, Facultad de Medicina Veterinaria y Zootecnia, Universidad Peruana Cayetano Heredia. Lima, Perú.

Presentado: $\quad 10 / 02 / 2010$ Aceptado: $\quad 23 / 07 / 2010$ Publicado online: $14 / 12 / 2010$

\author{
Alfonso Chavera C. ${ }^{1}$, Rufino Cabrera ${ }^{2,3}$, y Manuel Tantaleán ${ }^{4}$
}

\section{Resumen}

Se describen las lesiones pulmonares ocasionadas por el nematodo, Pseudalius inflexus (Rudolphi, 1808) Schneider, 1866 en dos especímenes, macho y hembra, de Phocoena spinipinnis Burmeisteir, 1865 "marsopa espinosa", capturados en aguas de la Reserva Nacional de Paracas, Perú. Macroscópicamente, los pulmones mostraron nódulos indurados superficiales de $0,5-2 \mathrm{~cm}$ de diámetro, donde al corte se encontraron nematodos rodeados por una severa reacción inflamatoria crónica y en los bronquios se observa que el extremo anterior de los parásitos obstruye la luz mientras que el extremo posterior se encuentra libre. Microscópicamente, los parásitos están en diferentes grados de degeneración, rodeados de un extenso exudado inflamatorio compuesto por eosinófilos, macrófagos, mononucleares, células gigantes multinucleadas con extenso tejido de granulación que infiltra el parénquima pulmonar, alvéolos distendidos y en algunos sectores destruidos. Además, se observa hiperplasia de mucosa bronquial, pared arterial con hiperplasia de capa muscular lisa, disminución del lumen. $P$. inflexus ocasiona una bronconeumonía crónica severa. Esta es la primera descripción de las lesiones pulmonares que produce $P$. inflexus en $P$. spinipinnis.

Palabras clave: Nematoda, Parasitos, Cetacea, Phocoena spinipinnis, Neumonía.

\section{Abstract}

Pulmonary lesions caused by the nematode Pseudalius inflexus (Rudolphi, 1808) Schneider, 1866; in two specimens, male and female of Burmeister's Porpoise (Phocoena spinipinnis Burmeisteir, 1865). The Cetaceans were captured in the Reserva Nacional de Paracas, Peru. Macroscopically, the lung shown superficial harden nodules of $52 \mathrm{~cm}$ of diameter and on the cut surface the nematodes were seen surrounded by severe chronic inflammatory reaction and the bronchium are occluded by the cranial part of these parasites and the caudal part remains free. Microscopically, the parasites are shown in different degree of degeneration and they are surrounded by severe inflammatory exudate composed of eosinophils, macrophages, mononuclear cells, multinuclear giant cells and dense granulation tissue scattered by the pulmonary parenchyma with distended and necrotic alveoli. Other lesions included hyperplasia of bronchium mucosa and hyperplasia of the arterial smooth muscle with luminal obliteration. Therefore, a severe eosinophilic granulomatous bronchopneumonia is caused by $P$. inflexus and is the first description of the parasitic pulmonary lesions in $P$. spinipinnis.

Keywords: Nematoda, Parasites, Cetacea, Phocoena spinipinnis, Pneumonia.

\section{Introducción}

El conocimiento de las lesiones que producen los parásitos en los cetáceos marinos, en especial los helmintos, es importante porque ocasionan graves enfermedades (Raga et al. 1997). Dentro de los helmintos, los nemátodes pulmonares de la familia Pseudaliidae son muy importantes porque ocasionan bronquitis y neumonía e incluso la muerte de fócidos y delfines tanto en poblaciones naturales (Geraci \& Aubin 1987) como en animales en cautiverio (Onderka 1989). Pseudalius inflexus (Rudolphi, 1809) es uno de los parásitos pulmonares más frecuentes de la marsopa Phocaena phocaena, cetáceo que habita las costas de Canadá, Estados Unidos (Margolis \& Arai 1987) y Reino Unido (Europa), donde las infecciones por esta especie ocasionan neumonía y es la segunda causa de muerte de este hospedero (Jepson et al. 1999).

Phocoena spinipinnis Burmeister, 1865, conocido como la marsopa negra o espinosa, y chancho marino en el Perú, es un cetáceo que habita las costas de América del Sur, desde el norte de Perú en el Océano Pacífico hasta el sur de Brasil en el Océano Atlántico. Se conoce muy poco sobre su biología y ecología y ha sido catalogada como insuficientemente conocida por la IUCN (2010). En la costa peruana, hasta la prohibición de su caza, P. spinipinnis fue uno de los mamíferos marinos capturados por pescadores artesanales, debido al consumo directo por la población (Arias 1996).

Torres et al. (1992) y Berón-Vera et al. (2008) han identificado algunos helmintos en los $P$. spinipinnis que habitan aguas chilenas y argentinas; sin embargo, no se conoce los efectos patológicos que producen en este cetáceo.

Entre los helmintos reportados para P. spinipinnis están los nemátodes Stenurus australis (Sarmiento \& Tantaleán, 1991), Halocercus sp. y P. inflexus, éste último de P. spinipinnis del litoral de Chimbote, departamento de Ancash (Tantaleán 1993) y de la Reserva Nacional de Paracas en Ica (Tantaleán \& Cabrera 1999). Existen pocos estudios sobre muerte y lesiones producidas por helmintos (Montes et al. 2004) o por otras causas (van Bresem et al. 2007) en cetáceos en el Perú; en particular no se conocían las lesiones patológicas que ocasiona $P$. inflexus en $P$. spinipinnis en las costas de América del Sur.

En el presente estudio se describen las lesiones pulmonares macroscópicas y microscópicas producidas por Pseudalius inflexus (Nematoda: Metastrongyloidea: Pseudaliidae) en Phocoena spinipinnis capturados, accidentalmente, por pesca artesanal en aguas de la Reserva Nacional de Paracas en el departamento de Ica, Perú. 


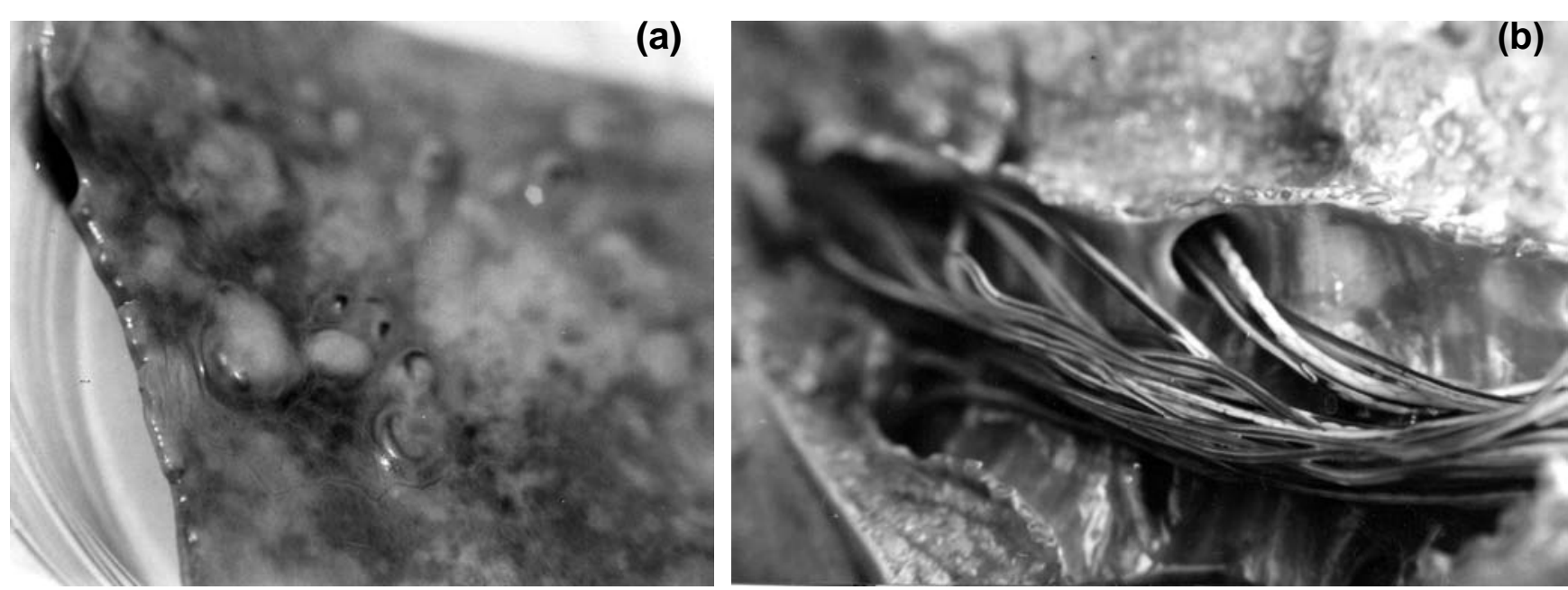

Figura 1. (a) Lesiones nodulares en la superficie del parénquima pulmonar de Phocaena spinipinnis producidas por Pseudalius inflexus. (b) Congestión de los bronquios por adultos de $P$. inflexus en $P$. spinipinnis. 5 X.

\section{Material y métodos}

Se examinaron pulmones de dos carcasas frescas de adultos (macho y hembra) de Phocoena spinipinnis, capturados, accidentalmente, el 8 de junio de 1994, y el 7 de mayo de 1997, por pescadores artesanales en el litoral de la Reserva Nacional de Paracas y desembarcados en el muelle pesquero de San Andrés, Pisco (13044’00”S - 76²13'29”W). La identificación de los hospederos se realizó en base a la fórmula dentaria y a las características morfológicas de acuerdo a Reyes et al. (1988) y Jefferson et al. (1993). Los procedimientos de la colecta, fijación e identificación de los helmintos fueron descritos por Tantaleán y Cabrera (1999).

Las áreas afectadas de los pulmones derecho e izquierdo de ambas marsopas se seccionaron y dividieron en varias porciones similares y fijaron en formol al 10\%, luego, fueron embebidas en parafina. Los cortes histológicos se realizaron a $4-6 \mu \mathrm{m}$ de grosor y se colorearon con Hematoxylina-Eosina y tricrómica de Masson (Humason 1967). Las observaciones, microfotografías y medidas se realizaron en un microscopio de campo claro con objetivos de $10 \mathrm{X}$ y $40 \mathrm{X}$.

Especímenes de $P$. inflexus estudiados se encuentran depositados en la Colección Helmintológica del Museo de Historia Natural de la Universidad Nacional Mayor de San Marcos (MUSM), Lima, Perú, con el No. 1534.

\section{Resultados}

Se encontraron, aproximadamente, 30 - 40 nemátodes en los bronquios, bronquiolos y vasos sanguíneos del lóbulo caudal pulmonar que fueron identificados como adultos de Pseudalius inflexus (Tantaleán \& Cabrera 1999).

Lesiones macroscópicas.- Los pulmones se encontraron congestionados, firmes, marmoleados formando múltiples lesiones nodulares induradas blanquecinas que coalescen y sobresalen en la superficie del parénquima pulmonar, miden entre 0,5 y $2 \mathrm{~cm}$ de diámetro (Fig. 1a). Al abrir los bronquios, se encontraron los extremos cefálicos de decenas de nemátodes vivos y algunos muertos rodeados por una densa cápsula fibrosa, en cambio, los extremos posteriores de color rojizo se dirigen hacia la tráquea (Fig. 1b). La longitud total de los nematodos machos es $13 \mathrm{~cm}$ en promedio y de las hembras $15 \mathrm{~cm}$. Los bronquios muestran una marcada reacción fibrótica inflamatoria que los rodea y se extiende hasta el parénquima pulmonar.

Lesiones microscópicas.- El pulmón ha perdido su citoarquitectura espongiforme; los septos alveolares se presentan muy engrosados, con abundante tejido conectivo denso y colágeno con áreas de hialinización (Fig. 2a) que ocupa gran parte de los espacios alveolares, aparece de un color azulado con la técnica de tricrómica de Masson. En algunas zonas del músculo liso se observa una sustancia pétrea de depósito, basófila, que sugiere la presencia de calcio.

Los bronquios mayores, ocupados por numerosos nemátodes, de cutícula gruesa queratinizada se rodea de un extenso exudado inflamatorio del hospedero compuesto por eosinófilos en variables grados de degeneración, linfocitos, macrófagos en fase de ingestión, células gigantes multinucleadas y bacterias bacilares basófilas con extensa proliferación de tejido conectivo fibroso y su mucosa revestida por un epitelio estratificado en exfoliación y la capa subyacente de músculo liso en marcada hiperplasia. Las arterias pulmonares presentan marcada hiperplasia de la musculatura lisa y disminución de sus lúmenes y la íntima con diversos grados de degeneración (Fig. 2b), los capilares alveolares con severa proliferación, congestión y formación de microtrombos. En otras áreas pulmonares se observan zonas de atelectasia y enfisema, rodeadas de tejido muscular liso muy prominente, hiperplásico que en su zona de contacto con el parásito contiene eosinófilos en variables grados de degeneración y macrófagos.

En otras zonas el parénquima pulmonar se observan cortes transversal y diagonal a diferentes niveles del nematode encapsulado resaltando la cutícula gruesa, los músculos de tipo polimiario y los cordones laterales (Fig. 2c).

\section{Discusión}

Esta es la primera descripción de bronconeumonía crónica producidas por el nematodo pulmonar $P$. inflexus en P. spinipinnis en aguas de América del Sur.

Pseudalius inflexus es el nematodo pulmonar más grande que parasita a $P$. phocoena de aguas británicas, noruegas y de Islandia, generalmente asociado a Torynurus convolutus; también se le ha implicado como causa de muerte (Baker \& Martin 1992, Gibson 

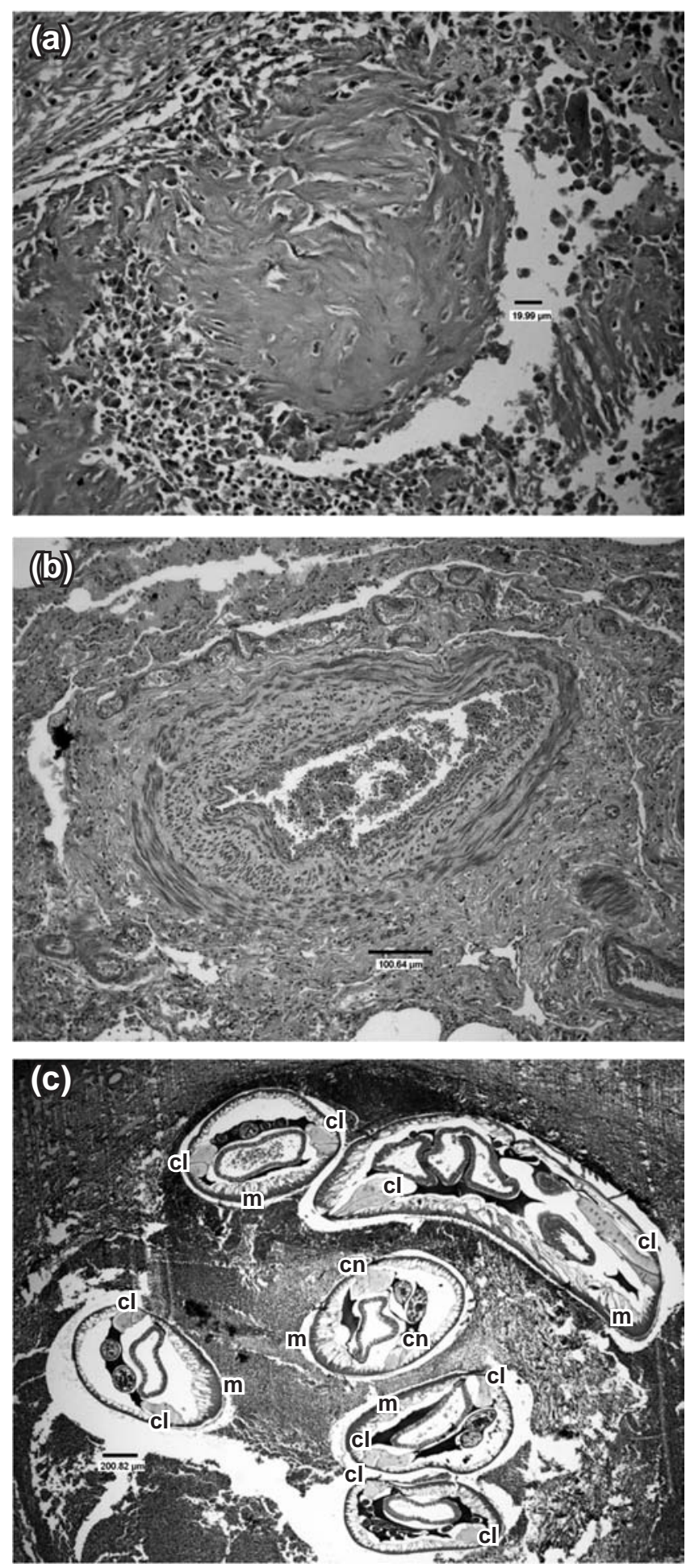

Figura 2. (a) Parénquima pulmonar con tejido conectivo, respuesta inflamatoria y destrucción del parénquima (bronquiolitis crónica). Coloración HE, 40 X. (b) Arteria pulmonar con hiperplasia marcada de la musculatura lisa. Nótese la disminución de la luz. Coloración HE, 10 X. (c) Parénquima pulmonar con corte transversal y diagonal del parásito encapsulado. Músculos $=\mathrm{m}$, cordones nerviosos $=\mathrm{cn}$ cordones laterales $=\mathrm{cl}$. Coloración HE, $10 \mathrm{X}$.

et al. 1998, Jepson et al. 2000). Se localiza en los bronquios y bronquiolos del pulmón de P. spinipinnis (Tantaleán \& Cabrera 1999) y de $P$. phocaena (Margolis \& Arai 1989); sin embargo, no se encontró en vasos pulmonares, corazón y tráquea, donde también habita la segunda especie (Baker \& Martin 1992, Gibson et al. 1998, Jepson et al. 2000, Siebert et al. 2006), quizás por el escaso número de animales examinados o por no haber revisado adecuadamente el corazón y número de secciones evaluadas histológicamente.

La densidad de infección de $P$. inflexus en $P$. spinipinnis, reportada en este trabajo, está dentro del rango encontrado en P. phocaena de aguas Europeas (Gibson et al. 1998, Slob et al. 1996) aunque el promedio es menor.

Las características macroscópicas e histopatológicas observadas en este trabajo son muy similares a las lesiones severas producidos en $P$. phocaena de las costas británicas (Jepson et al. 2000). Se ha sugerido que $P$. inflexus asociado a $T$. convolutus puede matar a $P$. phocoena en infecciones masivas (Baker y Martín 1992; Gibson et al. 1998) o por ruptura de la arteria pulmonar, bronquios y bronquiolos (Jepson et al. 2000). La arteritis observada en nuestro trabajo, con degeneración de la íntima y microtrombos en los capilares alveolares del pulmón de P. spinipinnis, corrobora la severidad de las lesiones que produce este nematodo; hallazgos patológicos similares se encontraron en infecciones por $P$. inflexus en el 17\% (34) ejemplares de $P$. phocaena en aguas británicas (Jepson et al. 2000).

Debido al tamaño, la localización y el número de $P$. inflexus en P. spinipinnis, se presume la congestión de los bronquios y septos, destrucción de los bronquiolos, lesión de vasos y del parénquima pulmonar de la marsopa, las que podrían ser causas de muerte natural; o debilitarlo y de esa forma, podrían ser atrapados en las redes de los pescadores artesanales. Es probable que los dos especímenes examinados hubieran varado y muerto debido a la neumonía crónica.

Las lesiones causados por $P$. inflexus en $P$. spinipinnis se asemeja a la neumonía verminosa que ocasiona Halocercus invaginatus en $P$. phocaena en las costas de los Estados Unidos de América, atribuyéndose el varamiento a este parásito (Dailey \& Strout 1978), aunque nosotros no observamos exudado de fibrina. Las lesiones provocadas por $P$. inflexus en $P$. spinipinnis son más severas que las provocadas por Torynurus convolutus en $P$. phocaena en aguas europeas (Baker \& Martin 1992), la presencia de trombos en las marsopas espinosas podrían deberse a la liberación de enzimas por el parásito.

Las lesiones peribronquiales alrededor del parásito podrían haber sido provocadas por la respuesta a sustancias liberadas por el poro excretor del nemátode, como se ha sugerido en las lesiones pulmonares de Phoca hispida, producidas por Otostrongylus circumlitus y Filaroides hispidus (Onderka 1989). La presencia de atelectasis y enfisema pulmonar por $P$. inflexus no habían sido descritas en estudios previos en otros cetáceos. Estas lesiones se habían observado en infecciones masivas por $H$. pingi en Neophocaena phocaenoides en las costas de Hong Kong (Parsons $\&$ Jefferson 2000).

En el diagnóstico diferencial de las lesiones histológicas de neumonía verminosa se debe considerar la infección por morbilivirus que puede ser descartada o confirmada por la prueba de inmunoperoxidasa (Jepson et al. 2000); a pesar de no haber sido reportada en los cetáceos de aguas peruanas, debe tenerse en cuenta.

Como $P$. inflexus ocasiona una bronconeumonía crónica severa en $P$. spinpinnis, podría tener un impacto negativo en la población natural de este cetáceo en las costas de América del Sur; por ello, se requieren de más estudios relacionados con los 
cambios patológicos provocados tanto por nemátodos pulmonares como de otros agentes, además es imprescindible aclarar el ciclo vital del parásito para plantear algún control o prevención de esta nematodiosis pulmonar.

\section{Agradecimiento}

Este estudio fue financiado parcialmente por PRO NATURALEZA, fundación peruana para la conservación de la naturaleza, gracias a la gestión del Blgo. Carlos Obando. También, agradecemos a Valerie Lounsbury del Nacional Aquarium, Baltimore (USA), Dr. Jhon Baker del Department of Veterinary Pathology, University of Liverpool, por la literatura proporcionada. Al Dr. Luis Gómez de la Facultad de Medicina Veterinaria de la Universidad Nacional Mayor de San Marcos por las facilidades brindadas para tomar las microfotografías.

\section{Literatura citada}

Arias, S. A. 1996. Informe sobre el estado de conocimiento y conservación de los mamíferos marinos del Perú. Inf. Prog. Inst. Mar Perú No 38:3-30.

Baker J.R. \& A.R. Martin. 1992. Causes of mortality and parasites and incidental lesions in harbour porpoise (Phocaena phocaena) from British waters. Vet. Rec. 130:554-558.

Berón-Vera B.; E. A. Crespo \& J. A. Raga. 2008. Parasites in stranded cetaceans of Patagonia. J. Parasitol. 94:946-948.

Dailey M. \& R. Strout. 1978. Parasites and associated pathology observed in cetaceans stranded along the Oregon coast. J. Wild. Dis. 14:503-511.

Geraci J.R. \& D.J. Aubin. 1987. Effects of parasites on marine mammals. Int. J. Parasitol. 17:407-414.

Gibson D.I., E.A. Harris, R.A. Bray, et al. 1998. A survey of the helminth parasites of cetaceans stranded on the coast of England and Wales during the period 1990-1994. J. Zool. London. 244:563-574.

Humason G. L. 1967. Animal tissue techniques, 2nd ed. WH Freeman and Company, San Francisco and London, 569 p.

Jefferson T.A., S. Leatherwood \& M.A. Webber. 1993. FAO species identification guide. Marine mammals of the world. FAO, Rome. 320 p.

Jepson P.D., P.M. Bennett, C.R. Allchin, et al. 1999. Investigating potencial associations between chronic exposure to polychlorinated biphenyls and infections disease mortality in harbour porpoises from England and Wales. Sci. Total. Environ. 243-244:339-348.

Jepson P.D., J.R. Baker, T. Kuiken, et al. 2000. Pulmonar pathology of harbour porpoises (Phocaena phocaena) stranded in England and Wales between 1990 and 1996. Vet. Rec. 21:721-727.

Margolis L. \& H.P. Arai. 1989. Parasites and marine mammals. In Synopsis of the parasites of vertebrates of Canada, M. J. Kenedy (ed). Alberta Agriculture Animal Division. Edmonton, 26 p.
Montes I.D., C.A. Chavera, M. van Bresem, et al. 2004. Descripción y evaluación anatómica de lesiones óseas cráneomandibulares en cetáceos odontocetos del mar peruano. Rev. Inv. Vet. Perú. 15:13-24.

Onderka D.K. 1989. Prevalence and pathology of nematode infections in the lungs of ringed seals (Phoca hispida) of the western Artic of Canada. J. Wild. Dis. 25:218-224.

Parsons E. C. M. \& T. A. Jefferson. 2000. Post-mortem investigations on stranded dolphins and porpoises from Hong Kong waters. J. Wild. Dis. 36:342-356.

Raga J.A., J.A. Balbuena, J. Aznar \& M. Fernández. 1997. The impact of parasites on marine mammals: a review. Parassitologia 39:293-296.

Reyes J.C., A.J. Read \& K. van Waerebeek. 1988. Clave para la identificación de los pequeños cetáceos más comunes en la costa peruana. Bol Lima No 57:93-95.

Sarmiento L. \& V.M. Tantaleán. 1991. Stenurus australis n. sp. (Nematodo: Pseudaliidae) de Phocaena spinipinnis (Burmeister, 1865) (Cetacea: Phocoenidae) del Perú. Publ. Mus. Hist. Nat. UNMSM Serie de Zoología (A) 36:1-4.

Siebert U., K. Tolley, G.A. Víkingsson, et al. 2006. Pathological findings in harbour porpoises (Phocaena phocaena) form Norwegian and Icelandic waters. J. Comp. Pathol. 134:134-142.

Slob C.M., W.M.L. Hendrikx, F.H.M. Borgsteede \& M. García Hartman. 1996. Lungworms in harbour porpoises (Phocaena phocaena) from Dutch waters. En: Proc. Third ECS Workshop on Cetacean Pathology, M. García Hartman (ed). European Cetacean Society Newsletter no. 37:35-40.

Tantaleán V. M. 1993. Algunos helmintos de mamíferos marinos del Perú y su importancia médica. Rev. Peru. Med. Trop. UNMSM. 7:67-71.

Tantaleán M. \& R. Cabrera. 1999. Algunos helmintos de la marsopa espinosa, Phocaena spinipinnis de la Reserva Nacional de Paracas, Perú. Parasitol. Día. 23:57-58.

Torres P.; J. A. Oporto; L. M. Brieva \& L. Escare. 1992. Gastrointestinal helminths of the cetaceans Phocoena spinipinnis (Burmeister, 1865) and Cephalorhynchus eutropia (Gray, 1846) from the southern coast of Chile. J. Wild. Dis. 28:313-315.

IUCN 2010. IUCN Red List of Threatened Species. Version 2010.4. $<$ www.iucnredlist.org $>$. Acceso 09/02/2010.

van Bresem M.F.; K. van Waerebeek; J. Reyes; et al. 2007. A preliminary overview of skin and skeletal disease and traumata in small cetaceans from South America waters. Lat. Am. J. Aquat. Mammal. 6(1):7-42.

van Waerebeek K.; J. C. Reyes \& J. Alfaro. 1993. Helminth of dusky dolphins Lagenorhynchus abscurus (Gray, 1828) from Peru. Aquatic Mammals 19:159-169. 